SAATKAMP, C. J.; MARTINS, A. A. O.; CARDOSO, I. S.; MOTA, L. S. A.; LIBERAL, M. A.; MAESTRI, C. Y. O.; SAATKAMP, J. G. S.; ALMEIDA, A. C.; FREIRE, A.; SENA, A. C. G.; SOUZA, J.; MAESTRI, R. P.

REF-ISSN1808-0804Vol.XI(1),01-10, 2014.

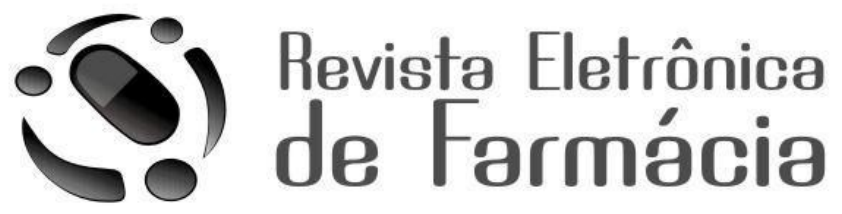

REF-ISSN1808-0804Vol.XI(1),01-10, 2014.

\title{
AVALIAÇÃO DO TEOR DE IODO PRESENTE NO SAL DE COZINHA COMERCIALIZADO NO MUNICÍPIO DE SANTARÉM PARÁ
}

\author{
EVALUATION OF IODINE CONTENT IN KITCHEN SALT COMMERCIALIZED \\ IN THE MUNICIPALITY OF SANTARÉM PARA
}

\begin{abstract}
Cassiano Junior Saatkamp ${ }^{1 *}$;Antônio Anastasis de Oliveira Martins ${ }^{2}$; Itamar dos Santos Cardoso ${ }^{3}$; Lucas da Silva Alho Mota ${ }^{4}$; Mauricio de Almeida Liberal ${ }^{5}$; Karen Cristini Yuni Ogawa Maestri ${ }^{6}$;Jamille Gomes dos Santos Saatkamp ;Arimar Chagas de Almeida ${ }^{8}$ Andréia Freire ${ }^{9}$;Ana Camila Garcia Sena ${ }^{10}$; Juarez Souza ${ }^{11}$; Régis PiloniMaestri ${ }^{12}$
\end{abstract}

\footnotetext{
${ }^{1}$ Mestrando em Bioengenharia; Instituto Esperança de Superior (IESPES); juniorsc7@yahoo.com.br

${ }^{2}$ Farmacêutico; Instituto Esperança de Superior (IESPES); antonio.martins@hotmail.com.br

${ }^{3}$ Farmacêutico; Instituto Esperança de Superior (IESPES); scardosoenil@gmail.com

${ }^{4}$ Farmacêutico; Instituto Esperança de Superior (IESPES); lucasalho@hotmail.com

${ }^{5}$ Mestrando em Bioengenharia; Instituto Esperança de Superior (IESPES);

mauricioliberal@hotmail.com

${ }^{6}$ Mestre em Doenças Tropicais; Instituto Esperança de Superior (IESPES);

karenmaestri@hotmail.com.br

${ }^{7}$ Especialista em Saúde da Família; Instituto Esperança de Superior (IESPES);

jamille santos@hotmail.com

${ }^{8}$ Especialista em Administração em Farmácia Hospitalar e Serviço de Saúde; Instituto Esperança de Superior (IESPES); arimaralmeidarc@yahoo.com.br

${ }^{9}$ Mestre em Gestão Ambiental; Instituto Esperança de Superior

(IESPES); andreiapalma @hotmail.com

${ }^{10}$ Mestranda em Gestão; Instituto Esperança de Superior

(IESPES);kamila sena@hotmail.com

${ }^{11}$ Doutorando em Doenças Tropicais; Instituto Esperança de Superior

(IESPES); souza644@hotmail.com

${ }^{12}$ Mestre em Doenças Tropicais; Instituto Esperança de Superior (IESPES); regismaestri@hotmail.com

* autor por correspondênciaInstituto: juniorsc7@yahoo.com.br

Esperança de Superior (IESPES), Av. Coaracy Nunes, 3315 - Caranazal - 68040-100

Santarém Pará-Brasil; Fone: (93) 3529-1760.
} 
SAATKAMP, C. J.; MARTINS, A. A. O.; CARDOSO, I. S.; MOTA, L. S. A.; LIBERAL, M. A.; MAESTRI, C. Y. O.; SAATKAMP, J. G. S.; ALMEIDA, A. C.; FREIRE, A.; SENA, A. C. G.; SOUZA, J.; MAESTRI, R. P.

REF-ISSN1808-0804Vol.XI(1),01-10, 2014.

RESUMO: A deficiência de iodo no organismo é considerada um grave problema de saúde pública, chegando a atingir cerca de 2 bilhões de pessoas, ou $35,2 \%$ da população global. A deficiência do mineral pode causar sérios problemas, afetandoprincipalmente grávidas e crianças, e se estender por toda a vida. Somente será considerado próprio para consumo humano o sal que contiver teor igual ou superior a 20 (vinte) até o limite máximo de 60 (sessenta) miligramas $(\mathrm{mg})$ de iodo por quilograma $(\mathrm{kg})$ de produto. Este trabalho objetivou avaliar o teor de iodo presente em amostras de sal de cozinha comercializados no município de Santarém - Pará. Foram coletadas aleatoriamente nove amostras de sal de cozinha em estabelecimentos que comercializam gêneros alimentícios no município de Santarém- Para. A técnica de quarteamento foi utilizada para se ter uma alíquota representativa, as amostras foram analisadas de acordo com o manual do Instituto Adolfo Lutz (2008). Os resultados mostram três amostras com teor de iodo fora de conformidade, sendo duas (02) amostras com valor acima do permitido e uma (01) abaixo do mínimo preconizado pelaANVISA. Em face da importância do iodo presente no sal, torna-se necessário um maior controle nos processos de fabricação pelas autoridades competentes. Os resultados obtidos neste trabalho evidenciaram a existência de desvio de qualidade em relação à concentração de iodo em amostras de sal comercializadas no município de Santarém - Pará, o que pode trazer problemas para a população.

PALAVRAS-CHAVE: Iodo, sal de cozinha, Bócio.

ABSTRACT: Body's iodine deficiency is considered a serious public health issue, reaching about 2 billion people, or $35.2 \%$ of the global population. The deficiency of this mineral in the body can cause serious problems, particularly affecting pregnant women and children, and extend throughout life (FREITAS et al., 2011; OMS, 2004). It will only be considered fit for human consumption the salt containingnot less than twenty (20) up to a maximum of sixty $(60)$ milligrams $(\mathrm{mg})$ of iodine per kilogram $(\mathrm{kg})$ of product (ANVISA, 2003).This study aimed at evaluating the content of iodine present in samples of table salt commercialized in the municipality of Santarém - Pará. Nine different samples of the product were randomly collected in stores that sell groceries in the city of Santarem - Para. The quartering technique was used to get a representative aliquot, the samples were analyzed according to the manual of the Adolfo Lutz (2008). The results show three samples with iodine content noncompliant, two (02) of which with value above the permitted and (01) below the minimum recommended by ANVISA (2003). Given the importance of iodine present in the kitchen salt, thus a stricter control in manufacturing processes by the competent authorities becomes necessary. The results of this study indicated the existence of quality deviation in relation to the concentration of iodine in salt samples sold in the municipality of Santarém - Pará, which can cause problems for the population.

KEY-WORDS:Iodine, kitchen salt, goiter .

\section{LISTA DE ABREVIAÇÕES}

ANVISA:Agência Nacional de Vigilância Sanitária

DDI: distúrbios por deficiência de Iodo

DNT: doença nodular da tireóide

mg: miligramas

$\mathrm{Kg}$ : quilograma 
SAATKAMP, C. J.; MARTINS, A. A. O.; CARDOSO, I. S.; MOTA, L. S. A.; LIBERAL, M. A.; MAESTRI, C. Y. O.; SAATKAMP, J. G. S.; ALMEIDA, A. C.; FREIRE, A.; SENA, A. C. G.; SOUZA, J.; MAESTRI, R. P.

REF-ISSN1808-0804Vol.XI(1),01-10, 2014.

OMS: Organização Mundial de Saúde

T3: triiodotironina

T4:tiroxina

TSH: hormônio estimulante da tireóide

\section{INTRODUÇÃO}

A Organização Mundial de Saúde (OMS) considera a deficiência de iodo como um grave problema de saúde pública e enfatiza que cerca de 2 bilhões de pessoas, ou $35,2 \%$ da população global, apresenta deficiência de iodo em suas necessidades diárias, sendo que as sequelas decorrentes dos Distúrbios por Deficiência de Iodo (DDI) podem se estender por toda a vida, principalmente em grávidas e crianças, sendo que simples políticas públicas podem evitar danos ao organismo devido esta deficiência, até a níveis cerebrais ${ }^{1,2} .0$ elemento iodo é considerado essencial e necessário às funções normais da tireóide, sua presença no organismo em níveis anormais pode causar distúrbios, como o bócio endêmico. Esta glândula sintetiza tiroxina (T4) e triiodotironina (T3) regulando a taxa do metabolismo e afetandoo aumento e a taxa funcional de muitos outros sistemas do corpo ${ }^{1}$. O iodo proveniente da dieta interfere diretamente na síntese dos hormônios tireoidianos. Após a absorção o iodo é captado pela tireóide, oxidado esobação enzimática liga-se à tireoglobulina para formação de T3 e T4. Em níveis séricos

os hormônios agem em receptores específicos nos tecidos, ativando a transcrição nuclear de grande número de genes, interferindo nos processos de crescimento e desenvolvimento, metabolismo de carboidratos e lipídeos, ações sobre o sistema nervoso central e os sistemas cardiovascular, respiratório, digestivo, endócrino e hematopoiético. A carência de iodo no organismo afeta a secreção dos hormônios promovendo alterações metabólicas. O excesso de iodo, por outro lado, pode aumentar os casos de tireoidite de Hashimoto e hipertireoidismo ${ }^{3}$.

A glândula tireóideé considerada uma glândula influenciada pelo eixo hipotálamo-hipófise, com a produção de seus principais hormônios são T3 Triiodotironina, representando $7 \%$ e T4 Tiroxina com produção de $93 \%$ do total, que tem a função de aumentar ou acelerar o metabolismo celular ${ }^{4}$. A ausência completa de secreção tireoidiana provoca a redução do metabolismo basal para $40 \%$ e $50 \%$ do normal, e o excesso extremo de secreção pode aumentar o tamanho da tireóide de $60 \%$ a $100 \%$. A secreção tireoidiana é controlada, principalmente, 
SAATKAMP, C. J.; MARTINS, A. A. O.; CARDOSO, I. S.; MOTA, L. S. A.; LIBERAL, M. A.; MAESTRI, C. Y. O.; SAATKAMP, J. G. S.; ALMEIDA, A. C.; FREIRE, A.; SENA, A. C. G.; SOUZA, J.; MAESTRI, R. P.

REF-ISSN1808-0804Vol.XI(1),01-10, 2014.

pelo hormônio estimulante da tireóide (TSH), secretado pela hipófise anterior ${ }^{5}$.

A primeira etapa na formação dos hormônios tireoidianos é o transporte de iodeto do sangue para as células e folículos glandulares da tireóide,ondea membrana basal das células tireoidianas bombeia ativamente iodeto para o interior da célula, sendo denominada de bomba de iodeto ${ }^{5}$.A doença nodular da tireóide (DNT) representa um aumento nodular clinicamente evidente da tireóide, caracterizado pelo crescimento excessivo e transformação estrutural e/ou funcional de uma ou várias áreas tireoidianas causada pela associação de fatores genéticos e ambientais, sendo a deficiência de iodo um importante fator ambiental.O tamanho da tireóide é inversamente proporcional à excreção de iodo $^{6}$. O agrupamento de todas as complicações supracitadas e morbidades associadas denominam-se de Moléstias Associadas à Carência de Iodo e/ou Distúrbios por Deficiência de Iodo, mostrando-se um sério obstáculo ao desenvolvimento social, econômico e mental da população em risco destacando o bócio endêmico como o mais incidente, representando um problema de saúde pública?

Quantificar os níveis de iodo no organismo é um parâmetro primordial para avaliação de como está sendo a ingestão do mineral. A quantificação pode ser realizada utilizando a urina do paciente, amostra de fácil coleta e de resultados precisos ${ }^{8}$.

Desde que foi determinada a obrigatoriedade da adição de iodo no sal, em 1953, o Ministério da Saúde realizou quatro pesquisas para quantificar a existência do bócio no Brasil com alunos de 6 a 14 anos de escolas públicas de várias regiões. A primeira, feita em 1955, detectou que $20,7 \%$ das crianças examinadas apresentaram a doença. Dezenove anos depois, outra pesquisa constatou um índice de $14,1 \%{ }^{9}$. O sal utilizado para o consumo humano "é um produto de consumo universal, razão pela qual, na quase totalidade dos países, é adotado como veículo para a adição de iodo, assumindo, assim, um importante papel no contexto social"10.

No Brasil, em março de 2003, a Agência Nacional de Vigilância Sanitária (ANVISA) em sua RDC no 130, de 26 de maio de 2003, e em seu Artigo $1^{\circ}$ estabeleceu que: "somente será considerado próprio para consumo humano o sal que contiver teor igual ou superior a 20 (vinte) até o limite máximo de 60 (sessenta) miligramas $(\mathrm{mg})$ de iodo por quilograma $(\mathrm{kg})$ de produto". Já no mês de abril de 2013, a ANVISA aprovou regulamento técnico que estabelece a nova teorização de iodo no sal de cozinha através da RDC $n^{\circ} 23$, publicada no mês de abril de 2013, a fim 
SAATKAMP, C. J.; MARTINS, A. A. O.; CARDOSO, I. S.; MOTA, L. S. A.; LIBERAL, M. A.; MAESTRI, C. Y. O.; SAATKAMP, J. G. S.; ALMEIDA, A. C.; FREIRE, A.; SENA, A. C. G.; SOUZA, J.; MAESTRI, R. P.

REF-ISSN1808-0804Vol.XI(1),01-10, 2014.

de erradicar efeitos nocivos à saúde, proposta esta, estudada desde o ano de 2011 com apoio parcial da população e maciço dos profissionais de saúde e dos órgãos competentes. Nesta norma, regularizou que o teor de iodo deverá estar igual ou superior de quinze (15) mg até o limite máximo de quarenta e cinco (45) $\mathrm{mg}$ por quilograma de produto, revogando a RDC publicada em maio de 2003.

O presente trabalho teve como objetivo avaliar o teor de iodo presente em amostras de sal de cozinha comercializadas no município de Santarém - Pará, avaliando se as concentrações encontradas estão de acordo com o que preconiza a ANVISA.

\section{MATERIAIS E MÉTODOS}

Foram coletadas aleatoriamente nove amostras de sal de cozinha em estabelecimentos que comercializam gêneros alimentícios no município de Santarém - Para. A técnica de quarteamento (Figura 1) foi utilizada para se ter uma alíquota representativa, as amostras foram analisadas de acordo com o manual do Instituto Adolfo Lutz ${ }^{12}$, na equação: O teor de iodo foi obtido a partir do seguinte fórmula: $V \quad x \quad x$ $105,8 / \mathrm{P}=\mathrm{mg}$ de Iodo/Kg de sal, onde, $\mathrm{V}=\mathrm{mL}$ da solução de tiossulfato de sódio 0,005 $M$ gasto na titulação; $f=$ fator da solução de tiossulfato de sódio $0,005 \mathrm{M}$ e $\mathrm{P}=\mathrm{n}^{\circ}$ de $\mathrm{g}$ da amostra.

Foram excluídas as amostras violadas, sem especificações de fabricação, como o produtor, tipo e informações técnicas. Também não se enquadraram nos critérios da pesquisa as amostras em acondicionamento inadequado, como armazenadas diretamente no chão, em locais úmidos ou que não estivessem nas prateleiras disponíveis para aquisição popular.

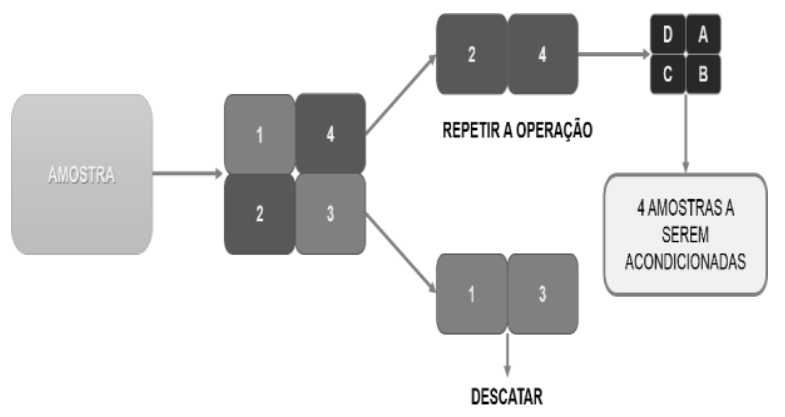

Figura 1 - Esquema de quarteamento

$\mathrm{Na}$ elaboração da tabulação dos dados coletados utilizamos o software Sigmaplot for Windows Version 10.0, criando um banco de dados dos testes realizados. Os resultados foram processados através de recursos da estatística descritiva, mediante utilização do software Excel (Microsoft Office for Windows $®$ - 2013)

\section{RESULTADOS E DISCUSSÃO}

Os resultados apresentados na Tabela 1 mostram o teor de Iodo 
SAATKAMP, C. J.; MARTINS, A. A. O.; CARDOSO, I. S.; MOTA, L. S. A.; LIBERAL, M. A.; MAESTRI, C. Y. O.; SAATKAMP, J. G. S.; ALMEIDA, A. C.; FREIRE, A.; SENA, A. C. G.; SOUZA, J.; MAESTRI, R. P.

REF-ISSN1808-0804Vol.XI(1),01-10, 2014.

presente no sal de cozinha analisado e desvio padrão das concentrações. Apresentando resultados

de conformidade para as amostras que apresentam concentração do mineral dentro do que é preconizado pela RDC130/2003(limite mínimo: 20 mg/Kg e

limite máximo: $60 \mathrm{mg} / \mathrm{Kg}$ ) e não conformidade para as amostras que apresentam valores diferentes dos préestabelecidos pela legislação, tanto pra mais quanto pra menos. Sendo que $67 \%$ das amostras estão de acordo com legislação e $33 \%$ não atendem as exigências.

Tabela 1-Resultados do ensaio para determinação do teor de iodo no sal de cozinha

\begin{tabular}{lcc}
\hline Amostras & $\begin{array}{c}\text { Teor de Iodo } \\
(\mathbf{m g} / \mathbf{K g}) \pm \mathbf{s}\end{array}$ & Resultados \\
\hline Amostra A & $37,74 \pm 1,22$ & Conforme \\
\hline Amostra B & $52,90 \pm 1,06$ & Conforme \\
Amostra C & $39,50 \pm 0,61$ & Conforme \\
Amostra D & $80,06 \pm 0,61$ & Não conforme \\
Amostra E & $47,96 \pm 0,61$ & Conforme \\
Amostra F & $4,94 \pm 1,22$ & Não conforme \\
Amostra G & $30,68 \pm 1,06$ & Conforme \\
Amostra H & $27,51 \pm 0,00$ & Conforme \\
Amostra I & $64,19 \pm 0,61$ & Não conforme \\
\hline
\end{tabular}

A Tabela 1, mostra os resultados das amostras, sua faixa de normalidade e amostras que apresentaram valores fora dos préestabelecidos.Valores demonstram que existem produtos que apresentam concentrações o dobro do estabelecido, enquanto observa-se amostras que possuem quantidades aquém dos valores estabelecidos pela legislação.

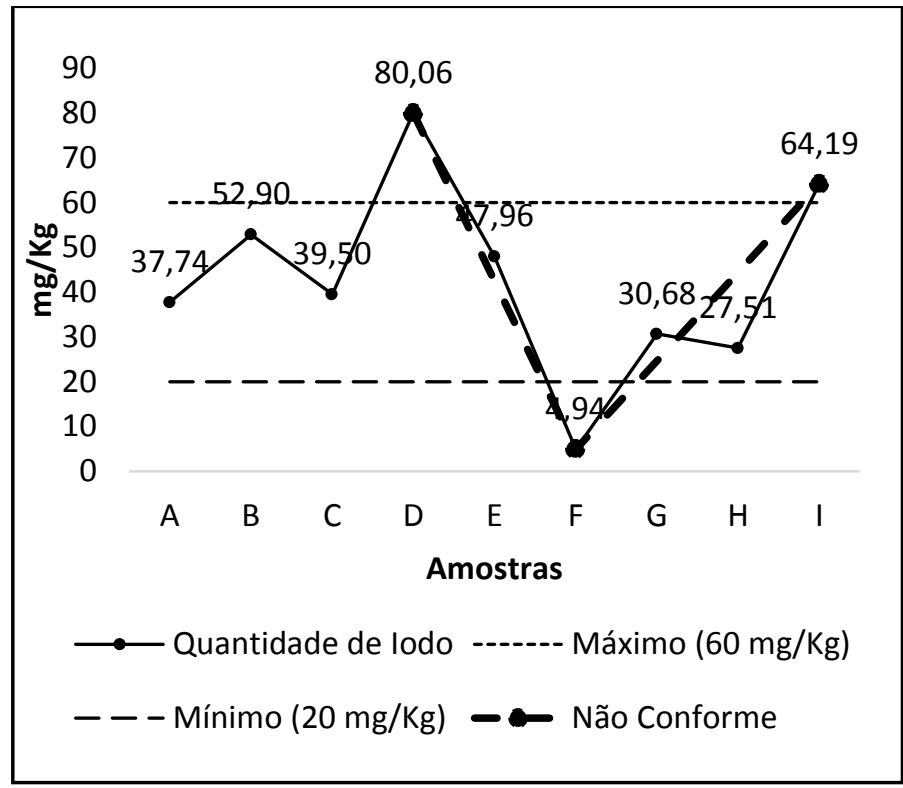

Figura 2 - Concentração de Iodo, faixa de normalidade e amostras não conformes.

Segundo Gama et al. (2010), na avaliação do teor de iodo do sal de cozinha comercializado em Palmas/TO, observou em seus estudos todas as amostras analisadas estavam em conformidade, ou seja, valores entre 20 a $60 \mathrm{mg}$ de iodo/Kg de sal.

$$
\text { Já Sant'Ana e Reges (2010), }
$$
avaliando o teor de iodo em 75 amostras de sal de cozinha e sal grosso, reporta que 10 amostras apresentaram concentrações irregulares de iodo com as concentrações preconizados pela legislação.

Em estudos realizados por Nimer e Silva (2002), com objetivo de estudar as possíveis associações entre a concentração de iodo na urina (iodúria) 
SAATKAMP, C. J.; MARTINS, A. A. O.; CARDOSO, I. S.; MOTA, L. S. A.; LIBERAL, M. A.; MAESTRI, C. Y. O.; SAATKAMP, J. G. S.; ALMEIDA, A. C.; FREIRE, A.; SENA, A. C. G.; SOUZA, J.; MAESTRI, R. P.

REF-ISSN1808-0804Vol.XI(1),01-10, 2014.

de escolares e a concentração de iodo no sal consumido por essas crianças, verificou-se que 161 estudantes consumiram sal com concentração de iodo abaixo da recomendação: 58,5\% do total. Dentre eles, 5,0\% apresentaram deficiência grave de iodo na urina, 6,2\%deficiência moderada, 25,5\% deficiência leve, e63,3\% apresentaram concentração normal de iodo na urina.

Em estudo realizado em 1994, com objetivo mapear as áreas de persistência da carência de iodo no Brasil, analisou a iodúria de 16.803 amostras de urina de escolares obtidas por coleta casual, em 401 municípios estudados. Foi observado deficiência em iodo em 85 municípios, sendo de grau moderado ( $\geq 25$ e $<50 \mu \mathrm{g} / \mathrm{L}$ ) em Cocos, na Bahia, e Almas,Arraias e Paraná, no Tocantins, e nos demais de grau leve(valores $\geq 50 \mathrm{e}<100 \mu \mathrm{g} / \mathrm{L}$ ). Em outros 35 municípios, a mediana dos valores foi normal, mas parcela significativa da população (mais de $10 \%$ das crianças) apresentou níveis de iodo inferiores a $25 \mu \mathrm{g} / \mathrm{L}$. Alguns fatores podem explicar essa variação, dentre eles um fator a ser considerado é que existindo um programa de suplementação alimentar de iodo, ocorre uma dispersão muito maior dos resultados relacionada ao grau de conscientização e ao poder aquisitivo da população ${ }^{16}$.
Anualmente, os brasileiros consomem aproximadamente um milhão de toneladas de sal, prevenindo uma das mais visíveis doenças derivadas da falta de iodo: o bócio. O monitoramento da qualidade do sal de cozinha está diretamente ligado ao controle dessa doença e de outros Distúrbios por Deficiências de Iodo (DDI), males causados pela ausência de iodo no organismo atingindo pessoas de todas as faixas etárias ${ }^{11}$. Falta de controle na concentração de iodo no sal consumido pela população, pode desencadear patologias de grande importância como retardo mental, pois atinge tanto o feto como o recém-nascido, prolongando-se pela fase escolar induzindo 0 baixo rendimento escolar, a dificuldade de adaptação sociocultural e a incapacidade relativa de trabalho na vida adulta, "a cognição compreende todos os processos mentais que nos permitem reconhecer, aprender, lembrar e conseguir trocar informações no ambiente em que vivemos $^{\prime 17}$.

Novas determinações foram adotadas em relação ao limite de Iodo presente no sal de cozinha, o teor deve estar igual ou superior de quinze (15) mg até o limite máximo de quarenta e cinco (45) mg por quilograma de $\mathrm{sal}^{18}$, com base nessa nova resolução, o numero de amostras em não conformidade subiria. 
SAATKAMP, C. J.; MARTINS, A. A. O.; CARDOSO, I. S.; MOTA, L. S. A.; LIBERAL, M. A.; MAESTRI, C. Y. O.; SAATKAMP, J. G. S.; ALMEIDA, A. C.; FREIRE, A.; SENA, A. C. G.; SOUZA, J.; MAESTRI, R. P.

REF-ISSN1808-0804Vol.XI(1),01-10, 2014.

\section{CONCLUSÃO}

\section{AGRADECIMENTO}

Em face da importância do teor de iodo presente no sal, torna-se necessário um maior controle nos processos de fabricação pelas autoridades competentes. Os resultados obtidos neste trabalho evidenciaram a existência de problemas em relação à concentração de iodo em amostras comercializadas, o que pode acarretar problemas de saúde aos consumidores, no caso a população em geral, haja visto que o sal é um produto consumido universalmente, e em muitas situações, o sal é uma das únicas fontes de iodo.

A Fundação Esperança,
mantenedora do Instituto Esperança de
Ensino Superior - IESPES pelo incentivo
e apoio para que essa pesquisa fosse
desenvolvida.
Aos Professores Marcos Moura
Gentil eFernando Monteiro D'Andrea,
pelas contribuições ao trabalho.

\section{REFERÊNCIAS}

1. DE FREITAS, GuttoRaffyson Silva et al. ANÁLISE DE IODATO EM SAIS DE COZINHA. Eclética Química, v. 36, n. 1, p. 93-109, 2011.

2. ORGANIZAÇÃO MUNDIAL DE SAÚDE - OMS. Nutrition: micronutrientdeficiencies. Disponível em:< http://www.who.int/nutrition/topics/idd/en/>. Acessado em: 09 outubro 2012.

3. LINS, Ana Maria da Silva Curado. Sistema Endócrino Hormônios da Tireoide. Disponível

em: <http://professor.ucg.br/SiteDocente/admin/arquivosUpload/5583/material/Tire\%C3 \%B3ide.pdf > . Acessado em 10 outubro 2012.

4. PEREIRA, Airton Vicente et al. Avaliação da Qualidade de Amostras Comerciais de Sal de Cozinha. Iniciação Científica Cesumar, v. 10, n. 2, p. 97-101, 2008.

5. GUYTON, Arthur Clifton; HALL, John E.; GUYTON, Arthur C. Tratado de fisiologiamédica. Elsevier, 2006.

6. GRAF, Hans. Doença nodular de tireóide; Thyroid nodular disease. Arq. bras.endocrinol. metab, v. 48, n. 1, p. 93-104, 2004. 
SAATKAMP, C. J.; MARTINS, A. A. O.; CARDOSO, I. S.; MOTA, L. S. A.; LIBERAL, M. A.; MAESTRI, C. Y. O.; SAATKAMP, J. G. S.; ALMEIDA, A. C.; FREIRE, A.; SENA, A. C. G.; SOUZA, J.; MAESTRI, R. P.

REF-ISSN1808-0804Vol.XI(1),01-10, 2014.

7. COSTA, Rosa M.; CARVALHO, Luis A. Experimentando um Ambiente Virtual com Pacientes Neuropsiquiátricos. QDLV GD, \&RQIHURQFLD, QWHUQDFLRQDO GH 7HFQRORJLDV GH, QIRUPDomR H \&RPXQLFDomR QD (GXFDomR, p. 529, 2001.

8. DUNN, JOHN T. et al. Two simple methods for measuring iodine in urine.Thyroid, v. 3, n. 2, p. 119-123, 1993.

9. AGÊNCIA NACIONAL DE VIGILÂNCIA SANITÁRIA. Informes Técnicos Institucionais. Revista de Saúde Pública. V. 34, P. 611 - 612, 2004.

10. INSTITUTO NACIONAL DE METROLOGIA, NORMALIZAÇÃO E QUALIDADE INDUSTRIAL - $\quad$ INMETRO. Sal refinado. Disponível em:< http://www.inmetro.gov.br/consumidor/produtos/sal.asp>. Acessado em: 09 outubro 2012.

11. . AGÊNCIA NACIONAL DE VIGILÂNCIA SANITÁRIA. Resolução no 130, de 26 de maio de 2003. Determina o limite máximo do teor de iodo exceda em três vezes o limite mínimo, face às características do beneficiamento do sal principalmente no que se refere à etapa de iodação. Diário Oficial da União, Poder Executivo, Brasília, DF, 2 mai. 2003.

12. Instituto Adolfo Lutz (São Paulo). Métodos físico-químicos para análise de alimentos

/coordenadores Odair Zenebon, NeusSadoccoPascuete Paulo Tiglea -- São Paulo:

Instituto Adolfo Lutz, 2008 p. 1020 (pag. 721)

13. GAMA, Jacqueline Christine Santos et al. AVALIAÇÃO DO TEOR DE IODO DO SAL DE COZINHA COMERCIALIZADO EM PALMAS-TO EVALUATION OF THE IODINE CONTENT OF SALT COMERCIALIZED IN PALMAS-TO.

14. SANTA'ANA, L.T.D.; REGES, I.S. Avaliação da qualidade do sal e tempero comercializados no estado do Tocantins, tomando como base o Programa Pró-Iodo. In: IV Simpósio de Engenharia, Ciência e Tecnologia de Alimentos, Foz do Iguaçu, 2010. Disponível em:< http://www.sovergs.com.br/site/higienistas/trabalhos/10298.pdf>. Acessado em: 05 maio 2013.

15. NIMER, Margarete; SILVA, Marcelo Eustáquio; OLIVEIRA, José Eduardo Dutra de. Associações entre iodo no sal e iodúria em escolares, Ouro Preto, MG. Rev Saúde Pública, v. 36, n. 4 , p. $500-4,2002$.

16. ESTEVES, Roberto Z. etal.Development of a semi-automated method for measuring urinary iodine and its application in epidemiological studies in Brazilian schoolchildren. Arquivos Brasileiros de Endocrinologia \& Metabologia, v. 51, n. 9, p. 1477-1484, 2007.

17. COSTA, Rosa M.; CARVALHO, Luis A. Experimentando um Ambiente Virtual com Pacientes Neuropsiquiátricos. QDLV GD, \&RQIHUrQFLD, QWHUQDFLRQDO GH 7HFQRORJLDV GH, QIRUPDomR H \&RPXQLFDomR QD (GXFDomR, p. 529, 2001.

18. AGÊNCIA NACIONAL DE VIGILÂNCIA SANITÁRIA. Resolução no 23, de 24 de abril de 2013. Dispõe sobre o teor de iodo no sal destinado ao consumo humano e dá outras providências. Diário Oficial da União, Poder Executivo, Brasília, DF, 24 abr. 2013. 
SAATKAMP, C. J.; MARTINS, A. A. O.; CARDOSO, I. S.; MOTA, L. S. A.; LIBERAL, M. A.; MAESTRI, C. Y. O.; SAATKAMP, J. G. S.; ALMEIDA, A. C.; FREIRE, A.; SENA, A. C. G.; SOUZA, J.; MAESTRI, R. P.

REF-ISSN1808-0804Vol.XI(1),01-10, 2014. 